\title{
Hierarchical large-scale elastic metamaterials for passive seismic wave mitigation
}

\author{
Marco Miniaci ${ }^{1,}{ }^{*}$, Nesrine Kherraz ${ }^{2}$, Charles Cröenne ${ }^{1}$, Matteo Mazzotti ${ }^{3}$, Maryam Morvaridi ${ }^{4}$, Antonio S. Gliozzi ${ }^{4}$, \\ Miguel Onorato ${ }^{5}$, Federico Bosia ${ }^{4}$, and Nicola Maria Pugno, \\ ${ }^{1}$ CNRS, Centrale Lille, ISEN, Univ. Lille, Univ. Valenciennes, UMR 8520 - IEMN, 59000 Lille, France \\ 2 Dipartimento di Fisica, Università degli Studi di Torino, Via Pietro Giuria 1, 10125 Torino, Italy \\ 3 Department of Mechanical Engineering, CU Boulder, 1111 Engineering Drive, UCB 427 Boulder, CO 80309, USA \\ ${ }^{4}$ Department of Applied Science and Technology, Politecnico di Torino, Corso Duca degli Abruzzi 24, 10124 Torino, Italy \\ ${ }^{5}$ Department of Physics, University of Torino, Via Pietro Giuria 1, 10125 Torino, Italy \\ ${ }^{6}$ University of Trento, Laboratory of Bio-Inspired, Bionic, Nano, Meta Materials \& Mechanics, Department of Civil, \\ Environmental and Mechanical Engineering, Via Mesiano 77, 38123 Trento, Italy
}

Received: 5 November 2020 / Accepted: 11 May 2021

\begin{abstract}
Large scale elastic metamaterials have recently attracted increasing interest in the scientific community for their potential as passive isolation structures for seismic waves. In particular, so-called "seismic shields" have been proposed for the protection of large areas where other isolation strategies (e.g. dampers) are not workable solutions. In this work, we investigate the feasibility of an innovative design based on hierarchical design of the unit cell, i.e. a structure with a self-similar geometry repeated at different scales. Results show how the introduction of hierarchy allows the conception of unit cells exhibiting reduced size with respect to the wavelength while maintaining the same or improved isolation efficiency at frequencies of interest for earthquake engineering. This allows to move closer to the practical realization of such seismic shields, where low-frequency operation and acceptable size are both essential characteristics for feasibility.
\end{abstract}

Keywords: Seismic phononic crystals / metamaterials / hierarchical organization / transient-dynamic analysis / vibration isolation

\section{Introduction}

Structural integrity and adherence to civil engineering requirements is of paramount importance in the construction of all types of buildings, especially when considering the protection from seismic hazards in large or strategic constructions, such as hospitals, nuclear sites, long span bridges, dams, etc. Seismic waves derive from the superposition of different types of elastic waves, including bulk waves (longitudinal and shear), and waves localized at the surface, also known as surface acoustic waves (SAWs) [1]. The latter are the most destructive, because of their reduced attenuation during propagation. Indeed, their slower decay with respect to bulk waves is the main cause for partial or even total collapse of buildings and structures

\footnotetext{
* e-mail: marco.miniaci@gmail.com; marco.miniaci@univlille.fr

${ }^{a}$ Also at: Queen Mary University of London, School of Engineering \& Materials Science, Mile End Road, London E1 4NS, UK.
}

during earthquakes [2]. As a consequence, the design of buildings and structures capable of withstanding large ground vibrations has been the focus of research for many decades, and a great number of isolating strategies has been proposed, including passive, active, hybrid and semi-active approaches [3-5]. However, despite these efforts, a commonly accepted method for the design of seismicresistant buildings has not yet been developed.

In 1999, Meseguer et al. [6] theoretically proposed for the first time to attenuate seismic SAWs through band gaps (BGs) obtained by drilling huge periodic holes in the ground. The dimensions of the holes proposed at that time were of the order of hundreds of meters in diameter and thousands of meters in depth. Such an extreme proposed solution derived from the measurement of the attenuation of SAWs in a scaled experiment of a marble quarry, performed in the $\mathrm{kHz}$ frequency regime. After this initial intuition, many years passed without substantial further investigation linking phononic crystals (PCs) and seismic wave attenuation, up to 2014, when Brûlé et al. [7] performed large scale experiments on meter-size periodic 
holes drilled in the ground. In this occasion the attenuation potential of periodic structures was measured in the $50 \mathrm{~Hz}$ frequency range and for bulk waves.

From then on, researchers have considered more and more types of PCs as a potential solution for reducing the displacement induced in buildings by seismic waves, including surface localized modes. Several designs have been proposed, based on both large-scale PCs and resonant structures $[8,9]$, trying to meet the stringent requirements of wave attenuation at extremely low frequencies (below $5 \mathrm{~Hz}$ ) [10], practical manufacturing designs [8], and other technological constraints. Among the resonator-based approaches, the most investigated consists in constructing pillar-like structures at the surface with the goal of redirecting seismic SAWs towards the bulk at a specific angle. This approach is also known in the literature as the "resonant metawedge" $[9,11]$. Its main drawback is the fact that the effect is rather narrow in frequency, since it relies on the resonance frequency of the resonators composing the system. Krödel et al. [12] showed that it was possible to enlarge the effective frequency range of these structures by using resonators with slightly different resonance frequencies. Other researchers explored the possibility of using buried resonators [13], or drilling periodic trench barriers [14,15], also including the possibility of saturated soil [16]. These methods also provided good attenuation, in larger frequency ranges, since their performance derives from Bragg scattering.

In the majority of these numerical studies, the analysis has been restricted to $2 \mathrm{D}$ models, assuming the geometry of the resonators/cavities to be infinite in one of the two horizontal directions. As a consequence, the full potential of the geometry of the resonators/cavities has been scarcely explored so far. Here, we overcome this limitation and, exploiting full 3D finite element modeling, we propose an innovative design strategy based on a hierarchical organization of large-scale PCs in the horizontal plane of the ground. We show that the introduction of hierarchy allows the conception of structures achieving isolation efficiency at lower frequencies if compared to the corresponding non hierarchical geometry, allowing to reduce the operating frequencies of the seismic barrier down to half their "non hierarchical" values, while leaving the unit cell size unaltered. Hierarchy also allows to manipulate the effective unit cell mechanical properties without the need of multiple materials with different mechanical characteristics. This is proved through band diagram calculation and transient dynamic analysis. Finally, the difference in terms of behavior adopting different boundary conditions (plate-like or in a half-space assumption) is also discussed.

\section{Description of the models}

We consider hierarchical structures, where hierarchy is understood in the sense that a representative unit cell comprises multiple arrangements of inhomogeneities at various scales. If the same arrangement occurs at every scale, the pattern is called self-similar [17]. In this work, we consider structures with self-similar cross-like cavities at two different hierarchical levels, as presented in Figure 1A and B, in a sandy soil matrix with Young's modulus $E_{\text {soil }}=20 \mathrm{MPa}$, Poisson's ratio $v_{\text {soil }}=0.3$, mass density $\rho_{\text {soil }}=1800 \mathrm{~kg} \cdot \mathrm{m}^{-3}$, as reported in Table 1 .

At first, we consider unit cells under the simplified assumption of free-free boundary conditions applied to the top and bottom surfaces, for the purpose of comparing the dynamic behavior of the standard and the hierarchical unit cells. Finite element models of the ordinary and hierarchical unit cells that compose the seismic hierarchical shield are shown in Figure $1 \mathrm{~A}$ and B. The ordinary unit cell consists of a cross-like cavity dug into the soil (Fig. 1A). The cross-like cavity divides the unit cell into two types of regions, referred in the following as (i) masses and (ii) connectors (see black arrows in Fig. 1A). The hierarchical unit cell is obtained by adding 4 additional cross-like cavities (see Fig. 1B and its inset), scaled down by a factor of 20, so as to fit in the regions connecting the four large square masses of the non-hierarchical structure. The corresponding geometrical parameters are: $A=5 \mathrm{~m}$, $B=0.9 A, C=0.2 A$ and $H=A$ for the ordinary unit cell and $a=A / 20, b=0.9 a, c=0.2 a$ and $H=A$ for the hierarchical one. Further geometrical details are reported in Table 2. Contrary to previous studies dealing with hierarchical organization, where the hierarchy was reproduced over the entire ordinary unit cell [17], here we introduce hierarchy only in the connectors, which are mainly responsible for the low frequency alteration of the BG [17]. The design strategy is implemented for only a single hierarchical level, but the procedure, in principle, can be recursively extended to $n$ hierarchical levels. The inclusions have been chosen in the shape of a cross-like cavity, due to its excellent capability to open low frequency large BGs $[18,19]$.

The band structures are computed for an infinite array of cells in the in-plane directions, i.e. with periodic conditions along the $x$ - and $y$-directions, and adopting standard Bloch-Floquet formalism, assuming a linear elastic behavior for the soil. The resulting eigenvalue problem is solved by varying the non-dimensional wavevector $\boldsymbol{k}^{*}$ along the irreducible path $[\Gamma-X]$, with $\Gamma \equiv(0,0)$ and $X(\equiv \pi / A, 0)$, with $A$ the lattice parameter using the commercial software Cosmol 5.5.

As a second step, we assume free boundary conditions applied to the top surface of the unit cell and of infinite halfspace at the bottom surface of the unit cell, which better reflects the condition of surface seismic wave propagation. This is modeled by adding a $8 \lambda$-long soil-like domain plus a $2 \lambda$-long PML region (where $\lambda$ is the wavelength) below the unit cell, as shown in Figure $2 \mathrm{~A}$, where for the sake of synthesis, only the hierarchical unit cell model is reported. Dispersion diagrams are calculated by applying the same aforementioned procedure.

Figure 2B reports a schematic representation of the finite element models used to perform dynamic transient calculations to evaluate and compare the attenuation performances of the ordinary and hierarchical unit cells. The model consists of a soil slab of length $L_{x}=300 \mathrm{~m}$, infinite in width in the $y$-direction (obtained by applying 

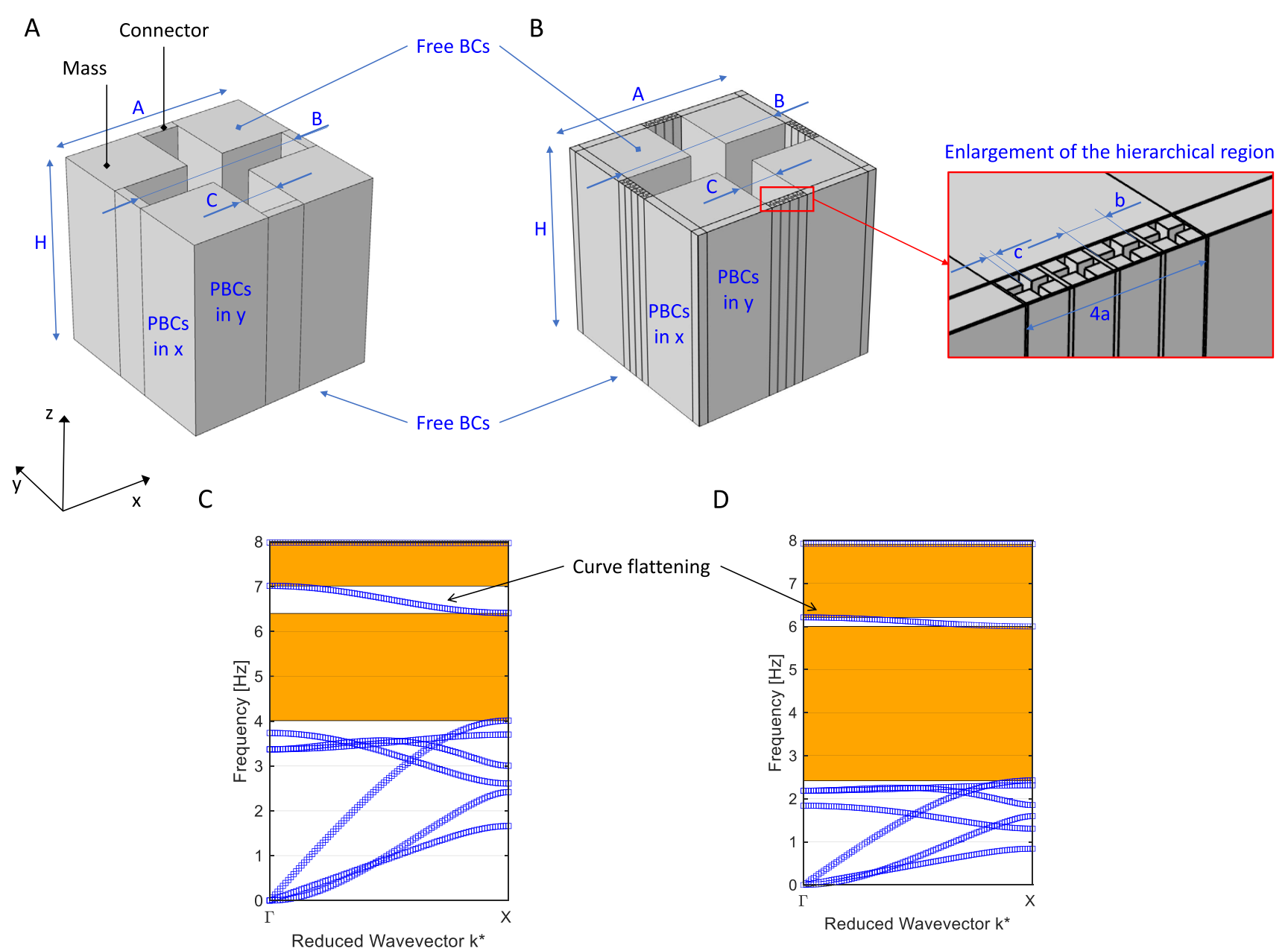

Fig. 1. Dispersion diagrams I - free/free assumption. (A) Schematic representation of the FE models for the ordinary and (B) hierarchical large-scale metamaterial (the inset provides an enlargement of the region where the second hierarchical level is introduced) under the assumption of free-free boundary conditions (BCs) applied to the top and bottom surfaces of the unit cell and periodic boundary conditions (PBCs) at lateral surfaces. Dispersion curves of the (C) ordinary and (D) hierarchical large-scale elastic metamaterial, showing a remarkable enhancement of the width of the BGs (orange rectangles) and their shift towards lower frequencies. Geometrical parameters of the unit cells for both ordinary and hierarchical structure are provided in the text.

Table 1. Material constants for the sandy soil model used in the simulations [8].

\begin{tabular}{llll}
\hline & $\rho_{0}\left[\mathrm{~kg} / \mathrm{m}^{3}\right]$ & $E[\mathrm{MPa}]$ & $\nu[/]$ \\
\hline Sandy soil & 1800 & 20 & 0.33 \\
\hline
\end{tabular}

Table 2. Geometrical parameters of the unit cells presented in Figure 1A,B and Figure 2A. All the given parameters are in $[\mathrm{m}]$.

\begin{tabular}{lllllll}
\hline$A$ & $B$ & $C$ & $H$ & $a$ & $b$ & $c$ \\
\hline 5 & 4.5 & 1.0 & 5 & 0.25 & 0.2250 & 0.05 \\
\hline
\end{tabular}

continuity conditions) and of height $L_{z}=8 \lambda+H ; 100 \mathrm{~m}$ ( $\lambda$ has been chosen according to the longest wavelength for the lowest frequency of $2 \mathrm{~Hz}$ ). Elastic waves are excited at the top-left edge of the model by means of a unitary imposed displacement in the $z$-direction (red arrows in Fig. 2B). Two input signals are considered: (i) a triangularlike excitation (left panel of Fig. 2C) and (ii) a 15-cycle Hanning window modulated sinusoidal tone burst excitation (right panel of Fig. 2C), exhibiting a rather broadband and narrow-band frequency content, respectively. Absorbing boundary (low-reflecting) conditions are imposed at the bottom and right edges of the model, using material data from the neighboring domain to develop a perfect impedance match for pressure and shear waves, to avoid unwanted reflections. Symmetry conditions are applied to the left edge of the model in order to be consistent with the type of induced excitation. 
A

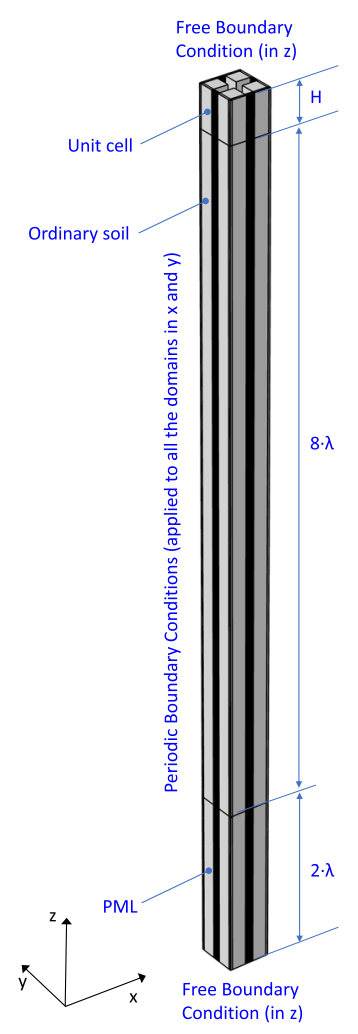

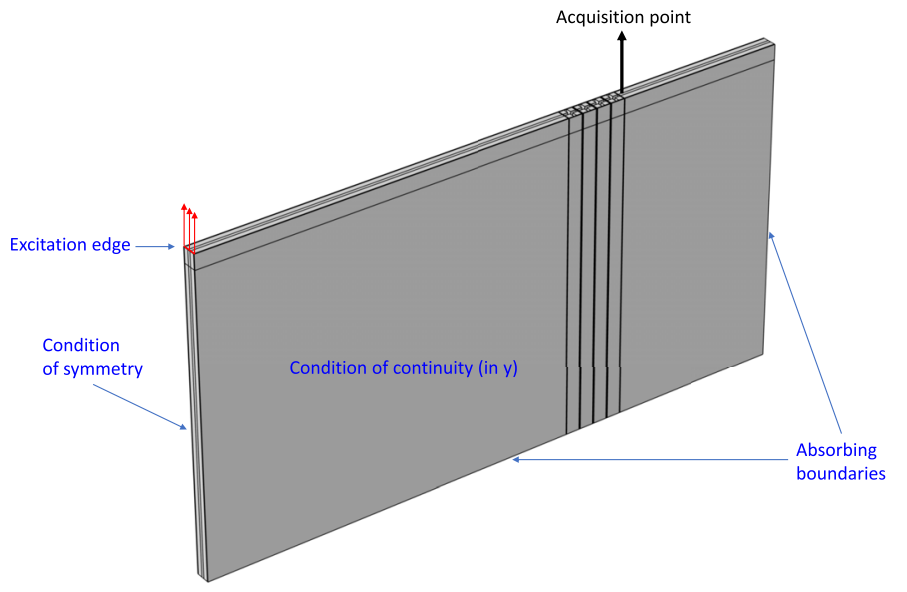

C
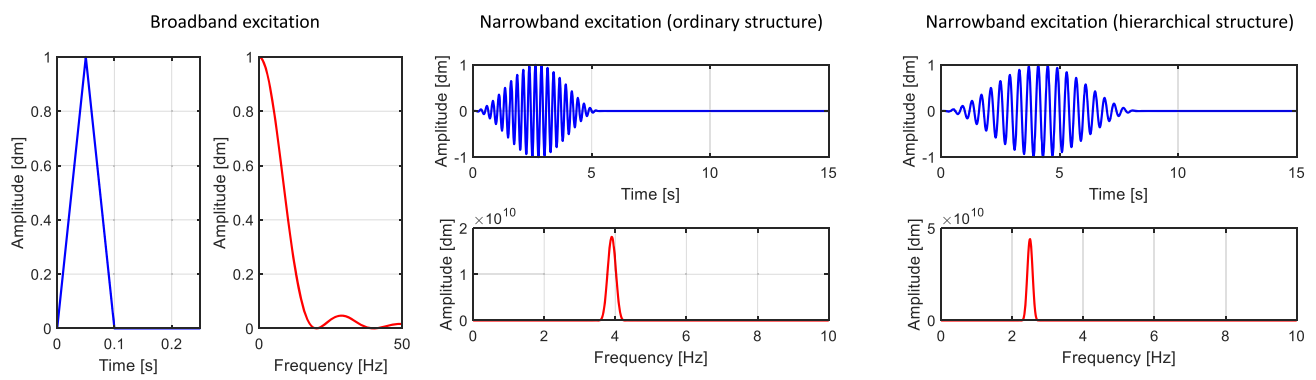

Fig. 2. Numerical models. (A) Schematic representation of the FE models used for the calculation of the dispersion curves localized at the surface. Free boundary conditions are applied to the top surface of the unit cell and a semi-infinite half-space is modeled through $8 \lambda$-long soil-like domain + a $2 \lambda$-long PML region. For the sake of synthesis, only the hierarchical unit cell is reported. (B) Schematic representation of the FE models used for the transient calculations. In this case, only the non hierarchical model is reported. (C) Time evolution and frequency content of the input signals used in simulations: (left panel) a triangular-like excitation and 21-cycle Hanning window modulated sinusoidal tone bursts centered at (central panel) $3.8 \mathrm{~Hz}$ and (right panel) $2.4 \mathrm{~Hz}$, respectively. The frequency spectrum clearly shows a rather broad-band and narrow-band frequency content, respectively. Displacement units are in decimeters [dm].

\section{Results}

Figure $1 \mathrm{C}$ and D reports the band structure of the ordinary and hierarchical unit cells, respectively, under the assumption of free-free boundary conditions applied to the top and bottom surfaces. We observe that hierarchy allows to obtain (i) a remarkable enhancement of the BG width (orange rectangles), as well as (ii) a BG shift towards lower frequencies. This twofold effect is a consequence of (i) the flattening of the dispersion curve (indicated by the black arrows in Fig. 1C,D) separating the two BGs of the ordinary structure and (ii) of a general decrease of the stiffness of the connectors, implying a downshift of the dispersion curves in the $[0-4] \mathrm{Hz}$ range.

Next, the SAW band structures for the ordinary and hierarchical unit cells are investigated and reported in Figure $3 \mathrm{~A}$ and $\mathrm{D}$ for the $[2-8] \mathrm{Hz}$ frequency range. Given the approximation of half-space condition as a $8 \lambda$-long soillike domain plus a $2 \lambda$-long PML region below the unit cell, a plethora of modes appear in the diagrams. However, the majority of them are not localized at the surface. To highlight this, curves are color-coded according to the parameter $p$, which is an indicator of the normalized center of energy distribution along the $z$-axis [20]:

$$
p=\left(1-\frac{\int_{V} E \cdot z d V}{H \int_{V} E d V}\right),
$$

having taken the integrals over the unit cell volume $V$, and where $E$ is the free elastic energy density and $H$ the total height of the unit cell. The parameter $p$ varies from 0 to 1 , where SAW-like modes are those with $p$ approaching 0 , as suggested by Graczyowki et al. [20]. We chose here $p \in[0,0.3]$. Once this sorting rule is applied, the diagrams can be simplified, selecting only SAW modes, as shown in Figure 3B and E. Some of the dispersion curves are not continuous along the whole Brillouin path $\Gamma-X$ because they undergo a transformation from true-SAWs to 
A
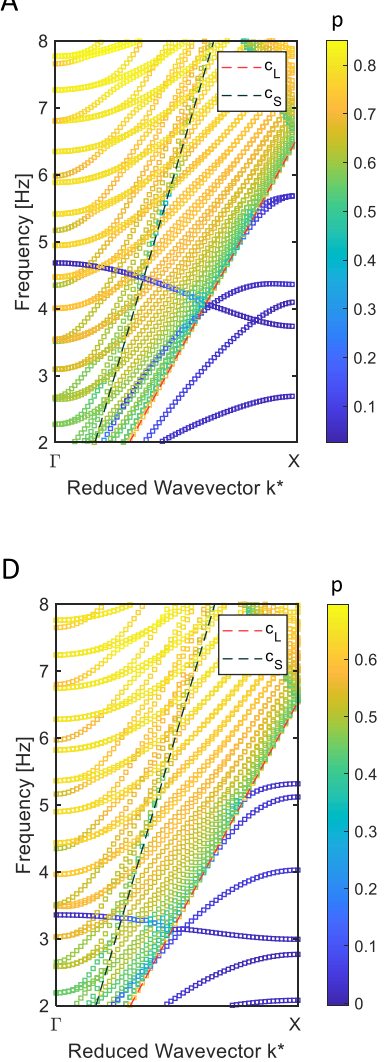



$\mathrm{E}$

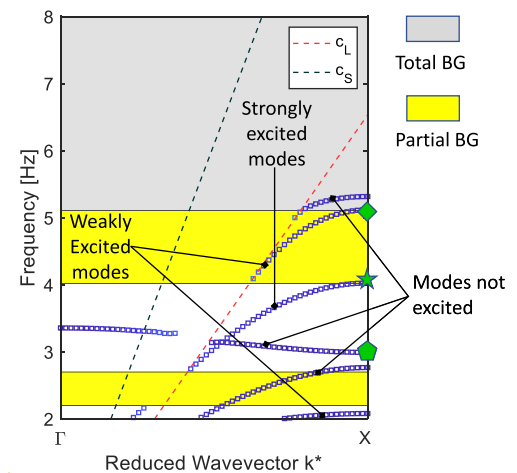

C
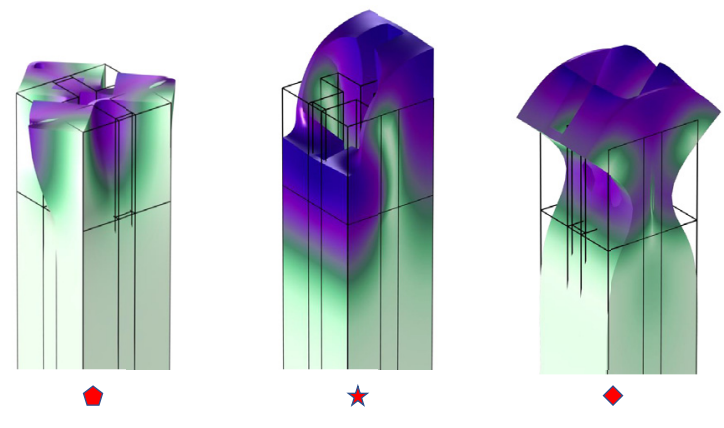

$\mathrm{F}$



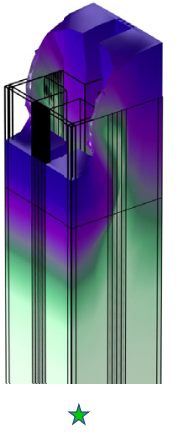

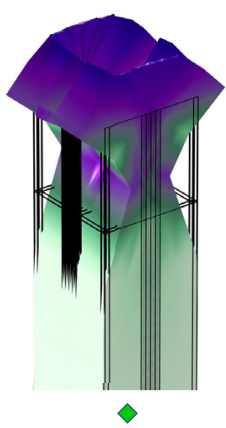

Fig. 3. Dispersion diagrams II - free/half-space assumption. Row dispersion diagrams deriving from the Bloch-Floquet calculations for the (A) ordinary and (D) hierarchical large-scale metamaterial, under the hypothesis of free boundary condition applied to the top surface of the unit cell and semi-infinite half-space modeled through $8 \lambda$-long soil-like domain + a $2 \lambda$-long PML region on the bottom surface of the unit cell. Curves are color-coded allowing to highlight modes localized at the surface $(p \leq 0.3)$ and those radiating in the bulk $(p>0.3)$. (B, E) Dispersion diagrams reporting only the modes localized at the surface for the dispersion diagrams reported in (A) and (D), respectively. Total and partial BGs are reported as grey and yellow rectangles, respectively. (C, F) Mode shapes with predominantly out-of-plane, in-plane and mixed polarization are indicated with corresponding symbols in the (B, E) band diagrams.

pseudo-SAWs (i.e., waves propagating with exponential attenuation, due to energy leakage into the bulk), as detailed in [20,21]. Figure $3 \mathrm{~A}$ and D also reports the longitudinal and shear bulk wave velocities as dashed black and red lines, respectively. This allows to evaluate the frequency of transition from surface to longitudinal/shear bulk waves and to make the distinction between true- and pseudo-SAWs.

Figure $3 \mathrm{~B}$ and $\mathrm{E}$ includes SAW-type modes with a predominantly out-of-plane polarization, with a weak outof-plane polarization, and mainly in-plane polarized (see mode shapes reported in Fig. 3C and F). Due to the nature of the excitation source considered in this study (forced out-of-plane displacement), we are interested in SAW-type modes with mainly out-of-plane polarization and we expect that while modes of the first type will be strongly excited in our system, those with a weak out-of-plane polarization will only provide a limited contribution to the vertical displacement, while those of the third type (in-plane polarized) will hardly be activated. As a consequence, we can identify two types of BGs that have been color-coded, using grey for those inhibiting the propagation of waves regardless of their polarization (in-plane, out-of-plane, mixed), and yellow to indicate BGs mainly inhibiting the propagation of in-plane polarized modes, referred to as total and partial BGs, respectively. Comparing the two band structures of the ordinary unit cell (Fig. 3B) and of the hierarchical one (Fig. 3E), we observe that the introduction of hierarchy also leads to (i) an enhancement of the BG width and (ii) a BG shift towards lower frequencies, as in the case of free-free boundary conditions. Specifically, the lowest BG is shifted from the $[3.6-4.0] \mathrm{Hz}$ range down to [2.2-2.7], while the highest one, starting at $5.68 \mathrm{~Hz}$ in the non-hierarchical structure, begins at $5.1 \mathrm{~Hz}$ in the hierarchical case. Notice that this is obtained while keeping the unit cell lattice parameter size unaltered. However, it is also important to remark that BGs are strongly reduced in width and appear in rather different frequency ranges, if compared to those deriving from the application of free-free boundary conditions. 


\section{Ordinary}

A
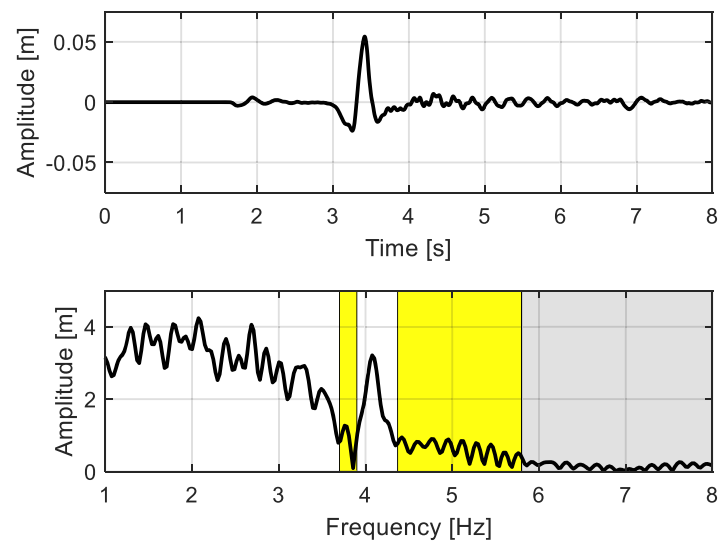

Hierarchical

B
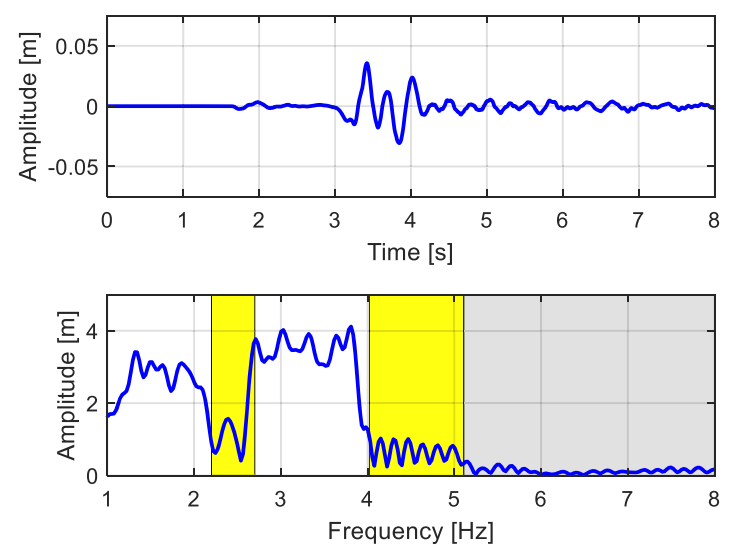

Total BG

Partial BG

Fig. 4. Time histories and Fourier transform. Out-of-plane time transient displacement and its Fourier transform for the broadband excitation for both the ordinary (A, black line) and hierarchical (B, blue line) structures. The signals are acquired immediately after the end of the periodic structure. The Fourier transforms of the signals allow to show the difference of the two responses in terms of frequency content, showing the ability of the hierarchical unit cell to provide large attenuation in the [2.2-2.7] $\mathrm{Hz}$ frequency range and above $5.1 \mathrm{~Hz}$, in good agreement with the dispersion diagrams reported in Figure 3B and E. BGs calculated in Figure 3B and $\mathrm{E}$ are superimposed onto each frequency response, to visually highlight how the frequency responses matches the dispersion diagrams.

The confirmation of the lowest BG opening/shift is then verified through a transient numerical simulation of wave propagation conducted on a finite size domain comprising a sandy soil block and 4 unit cells disposed in the $x$-direction, as shown in Figure 2B. Elastic waves are excited at the top-left edge of the model by means of a unitary imposed displacement in the $z$-direction (red arrows in Fig. 2B), according to the time-histories reported in Figure 2C of Section 2. Such pulses have been chosen according to the band structure reported in Figure 3B and $\mathrm{E}$ and to highlight the filtering capabilities of the designed hierarchical large scale PC compared with the nonhierarchical one. In both excitation cases, transient explicit simulations have been run for $T=8 \mathrm{~s}$ in order to allow the wave coming from the left side to reach the right end of the simulation domain.

The out-of-plane time transient displacements for both types of excitation are recorded at the end of the periodic structure (see Fig. 2B) and Fourier transformed to highlight the differences of the two responses for the ordinary and hierarchical large-scale PCs both in the time and frequency domains. Figure $4 \mathrm{~A}$ reports the displacement along the $z$-direction, as well as its frequency content (Fig. 4B) for the excitation case of the triangular pulse reported in Figure 2C, characterized by a broad-band frequency content. The strongest achieved attenuation is shifted from slightly below $6 \mathrm{~Hz}$ for the ordinary structure to $5.1 \mathrm{~Hz}$ in the hierarchical case (corresponding to the beginning of the total BG). Examining the frequency content of the transmitted displacement field at lower frequencies, it emerges that the hierarchical case is also capable of providing large attenuation in the $[2.2-2.7] \mathrm{Hz}$ frequency regime, in good agreement with the dispersion diagrams reported in Figure 3B and E. The slightly higher amplitude level observed in the partial BG frequency regions (i.e., those covered by the yellow rectangles) derives from modes that, although mainly in-plane polarized, still exhibit some out-of-plane displacement components. This is in good agreement with the two different dispersion diagrams and clearly confirms the possibility of using hierarchical unit cell organization to downshift stop-band behavior.

To gain further insight into this behavior, the timedomain simulations are repeated, this time with the previously defined narrow band signal (see Sect. 2), with modulations centered at 3.8 and $2.4 \mathrm{~Hz}$ for the nonhierarchical and hierarchical cases, respectively. Figure 5 shows the instantaneous out-of-plane displacement fields at specific times, namely before and after the wave has reached the periodic structures, for both the nonhierarchical and hierarchical cases. The wave pulse is strongly reflected in both cases. However, from a quantitative point of view, the maximum recorded displacements with respect to a model where the surface wave propagates freely (i.e. without any PC) are almost 8 and 3 times larger for the non-hierarchical and hierarchical cases, respectively. Note that part of the attenuation efficiency difference between the two cases is directly related to the fact that the hierarchical structure is evaluated at a lower frequency, namely $2 \mathrm{~Hz}$ instead of 4.

\section{Conclusion}

This study contributes to the conception of a new generation of earthquake-proof barriers capable of protecting sensitive 

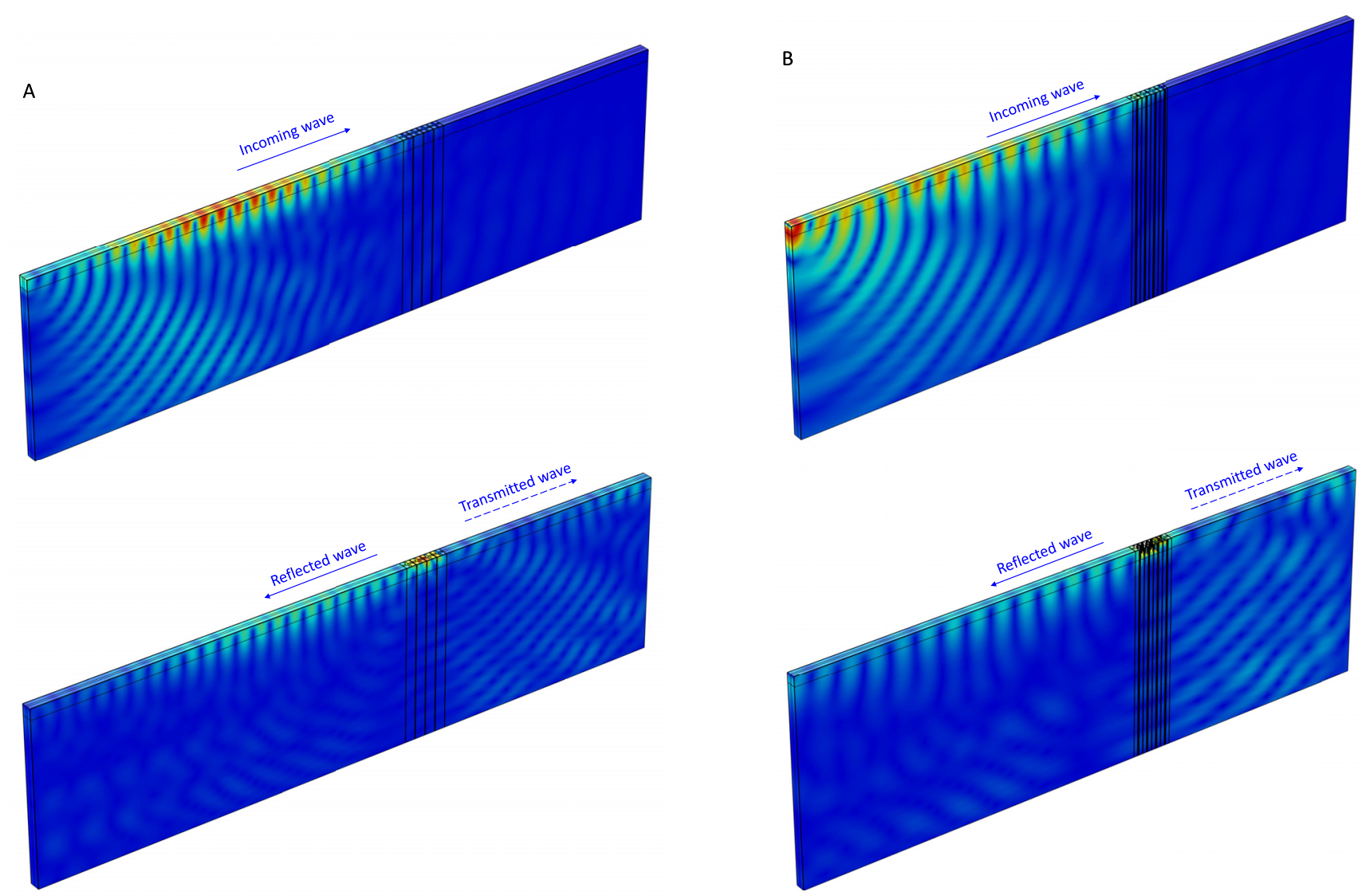

Fig. 5. Full wavefield reconstruction. Out-of-plane full wavefield for the (A) ordinary and (B) hierarchical seismic barrier at two different time steps: before (top panels) and after (bottom panels) the wave has reached the periodic structure.

or strategic structures, introducing the concept of hierarchical design. We have investigated the effect of hierarchy on dispersion diagrams and verified results through time-transient simulations, and highlighted the possibility of realizing unit cells capable of opening frequency BGs below $5 \mathrm{~Hz}$, despite being similar in size to previous designs. Results prove the strategy to be practical for civil structures.

MaMi has received funding from the European Union's Horizon 2020 research and innovation programme under the Marie Sklodowska Curie grant agreement N. 754364. NK, FB, ASG, MO have received funding from the project "Metapp" (No. CSTO160004) funded by Fondazione San Paolo. MaMi, MaMO, FB, ASG and NMP are funded by the European Union's Horizon 2020 FET Open ("Boheme") under grant agreement No. 863179 .

\section{References}

1. I. Viktorov, Rayleigh and Lamb Waves (1967)

2. R. Villaverde, Fundamental Concepts of Earthquake Engineering (CRC Press, 2009)
3. S.K. Duggal, Earthquake Resistant Design of Structures (Oxford University Press, New Delhi, 2007)

4. B. Spencer, Jr., S. Nagarajaiah, State of the art of structural control, J. Struct. Eng. 129, 845 (2003)

5. T. Datta, A state-of-the-art review on active control of structures, ISET J. Earthquake Technol. 40, 430 (2003)

6. F. Meseguer, M. Holgado, D. Caballero, N. Benaches, J. Sanchez-Dehesa, C. López, J. Llinares, Rayleigh-wave attenuation by a semi-innite two-dimensional elasticband-gap crystal, Phys. Rev. B 59, 12169 (1999)

7. S. Brûlé, E. Javelaud, S. Enoch, S. Guenneau, Experiments on seismic metamaterials: molding surface waves, Phys. Rev. Lett. 112, 133901 (2014)

8. M. Miniaci, A. Krushynska, F. Bosia, N.M. Pugno, Large scale mechanical metamaterials as seismic shields, New J. Phys. 18, 083041 (2016)

9. A. Colombi, D. Colquitt, P. Roux, S. Guenneau, R.V. Craster, A seismic metamaterial: The resonant metawedge, Sci. Rep. 6, 27717 (2016)

10. Y. Achaoui, T. Antonakakis, S. Brûlé, R. Craster, S. Enoch, S. Guenneau, Clamped seismic metamaterials: ultra-low frequency stop bands, New J. Phys. 19, 063022 (2017)

11. A. Colombi, P. Roux, S. Guenneau, P. Gueguen, R.V. Craster, Forests as a natural seismic metamaterial: Rayleigh wave bandgaps induced by local resonances, Sci. Rep. 6, 19238 (2016) 
12. S. Krödel, N. Thomé, C. Daraio, Wide band-gap seismic metastructures, Extreme Mech. Lett. 4, 111 (2015)

13. A. Palermo, S. Krödel, A. Marzani, C. Daraio, Seismic surface waves attenuation by buried resonators, in 2016 10th International congress on advanced electromagnetic materials in microwaves and optics (metamaterials) (IEEE, 2016) pp. $271-273$

14. J. Huang, Z. Shi, Attenuation zones of periodic pile barriers and its application in vibration reduction for plane waves, J. Sound Vib. 332, 4423 (2013)

15. X. Pu, Z. Shi, Broadband surface wave attenuation in periodic trench barriers, J. Sound Vib. 468, 115130 (2020)

16. G. Shi, G.-Y. Gao, Three-dimensional analysis of a row of piles as passive barriers in saturated soil, J. Vib. Eng. 23, 546 (2010)

17. M. Miniaci, A. Krushynska, A.S. Gliozzi, N. Kherraz, F. Bosia, N.M. Pugno, Design and fabrication of bioinspired hierarchical dissipative elastic metamaterials, Phys. Rev. Appl. 10, 024012 (2018)

18. M. Miniaci, A. Marzani, N. Testoni, L. De Marchi, Complete band gaps in a polyvinyl chloride (pvc) phononic plate with cross-like holes: numerical design and experimental verication, Ultrasonics 56, 251 (2015)

19. M. Miniaci, M. Mazzotti, M. Radzieński, N. Kherraz, P. Kudela, W. Ostachowicz, B. Morvan, F. Bosia, N.M. Pugno, Experimental observation of a large low-frequency band gap in a polymer waveguide, Front. Mater. 5, 8 (2018)

20. B. Graczykowski, F. Alzina, J. Gomis-Bresco, C. Sotomayor Torres, Finite element analysis of true and pseudo surface acoustic waves in one-dimensional phononic crystals, J. Appl. Phys. 119, 025308 (2016)

21. M. Mazzotti, M. Miniaci, I. Bartoli, Band structure analysis of leaky bloch waves in $2 \mathrm{~d}$ phononic crystal plates, Ultrasonics 74, $140(2017)$

Cite this article as: Marco Miniaci, Nesrine Kherraz, Charles Cröenne, Matteo Mazzotti, Maryam Morvaridi, Antonio S. Gliozzi, Miguel Onorato, Federico Bosia, Nicola Maria Pugno, Hierarchical large-scale elastic metamaterials for passive seismic wave mitigation, EPJ Appl. Metamat. 8, 14 (2021) 